\section{Liquid Crystalline Samples: Application to Macromolecular Structure Determination}

\author{
Ad Bax, James J. Chou, and Benjamin E. Ramirez \\ Laboratory of Chemical Physics, National Institute of Diabetes, and \\ Digestive and Kidney Diseases, National Institutes of Health, \\ Bethesda, MD, USA
}

\section{Introduction}

2 Theoretical Considerations

3 Measurement of Dipolar Couplings

4 Alignment Media for Macromolecules

5 Relation between Alignment and Shape

6 Structure Validation

7 Concluding Remarks

8 Related Articles in Volumes 1-8

9 References

1

\section{INTRODUCTION}

NMR spectra of small molecules dissolved in organic liquid crystalline media permit measurement of large numbers of intramolecular dipolar couplings that carry very precise information on molecular structure. ${ }^{1,2}$ However, for molecules with more than half a dozen protons, the strong degree of solute alignment obtained in such media typically results in a vast number of large, non-first-order splittings that make these spectra difficult to analyze. On the other hand, very weak alignment, caused by a molecule's own magnetic susceptibility anisotropy, also gives rise to measurable dipolar couplings. $\mathbf{3 , 4}$ However, typically only the largest couplings are detectable and manifest themselves as small, field-dependent changes in the corresponding $J$ splittings. The advantages of such ultra-weak alignment are that the NMR spectrum retains its regular high-resolution simplicity and that, owing to the extremely weak magnetic forces involved, it does not perturb the structure of the molecule.

Tolman et al..$^{\mathbf{5}}$ demonstrated that small dipolar couplings, on the order of a few hertz, could be measured for the backbone amides in paramagnetic myoglobin, and that these data correlate well with values expected on the basis of its crystal structure. Tjandra et al. subsequently measured a substantial number of very small ${ }^{15} \mathrm{~N}-{ }^{1} \mathrm{H}$ and ${ }^{13} \mathrm{C}^{\alpha}-{ }^{1} \mathrm{H}^{\alpha}$ dipolar couplings in a diamagnetic protein-DNA complex and demonstrated that incorporation of these data as restraints in the structure calculation yields a remarkable improvement in both local and global geometry. ${ }^{6}$ In particular, by defining the relative orientation of structural elements in a macromolecule with few interconnecting NOEs, these dipolar restraints solve a long-standing problem.

General applicability of the weak alignment approach greatly increased when the suitability of very dilute liquid crystalline media for this purpose was discovered. ${ }^{7-14}$ The macromolecules are dispersed in the aqueous phase that separates the highly ordered liquid crystalline particles, which have a spacing much larger than the diameter of the molecule under study. Despite the typically rather large macroscopic viscosity of such samples, the ordered particles are spaced by essentially pure water, and usually no adverse effect on rotational diffusion is therefore observed. ${ }^{7}$ However, exceptions to this rule can occur in cases where alignment is dominated by attractive electrostatic interactions between the nematogen and the solute. ${ }^{15}$ As discussed in Section 4.6, an alternate way for inducing weak alignment relies on axially compressed or stretched polyacrylamide gels. From a theoretical perspective, alignment in gels and in dilute lyotropic liquid crystalline suspensions are very similar.

The ability to tune the degree of macromolecular alignment over more than an order of magnitude, usually in the range from $3 \times 10^{-4}$ to $3 \times 10^{-3}$, makes it possible to measure a whole plethora of short-range dipolar interactions, including those between directly bonded ${ }^{1} \mathrm{H}-{ }^{13} \mathrm{C},{ }^{1} \mathrm{H}-{ }^{15} \mathrm{~N},{ }^{13} \mathrm{C}-{ }^{13} \mathrm{C}$ and ${ }^{13} \mathrm{C}-{ }^{15} \mathrm{~N}$ pairs, and also between spatially proximate nonbonded ${ }^{1} \mathrm{H}-{ }^{1} \mathrm{H},{ }^{1} \mathrm{H}-{ }^{13} \mathrm{C}$ and ${ }^{1} \mathrm{H}-{ }^{15} \mathrm{~N}$ spin pairs. ${ }^{8,16-18}$ The measurement and application of dipolar couplings and CSA effects in weakly oriented macromolecules has already proven to be extremely useful in biomolecular structural studies. Several reviews have appeared on various aspects of this work, ${ }^{19-22}$ and further developments in this area of research are occurring at a very rapid pace. Even at present, a comprehensive review of this area would fill hundreds of pages, and is therefore well beyond the scope of this article. Instead, the relation between this work and conventional liquid crystal NMR will be highlighted, as well as illustrating some of the advantageous features of the weak ordering approach.

\section{THEORETICAL CONSIDERATIONS}

Although standard theory described for liquid crystals elsewhere in this encyclopedia is directly applicable to the case of very weakly ordered macromolecules, the early applications of the weak ordering technology derive from the magneticsusceptibility induced alignment. As a result, the terminology differs somewhat and will be briefly reviewed below.

\subsection{Dipolar Couplings in Aligned Macromolecules}

The relation between the internuclear vector and the dipolar coupling between two spins, $A$ and $B$ can be found in numerous textbooks. For the purpose of deriving the resonance frequencies (i.e., dipolar splittings) only the $z$ component of the local field of one nuclear dipole at the position of the second nucleus is relevant (secular approximation):

$$
H_{d d}=D_{\max }^{A B}\left\langle I_{A z} I_{B z}\left(3 \cos ^{2} \zeta-1\right)\right\rangle
$$

where the \langle\rangle brackets refer to the time or ensemble average, which are equivalent for isotropic and liquid crystalline 
solutions, $\zeta$ is the angle between the $A-B$ internuclear vector and the magnetic field, and

$$
D_{\max }^{A B}=\frac{-\mu_{\mathrm{o}}(H / 2 \pi) \gamma_{A} \gamma_{B}}{\left(4 \pi^{2} r_{A B}^{3}\right)}
$$

is the static dipolar coupling in SI units $(21.7 \mathrm{kHz}$ for $\mathrm{H}-\mathrm{N}$ pairs, assuming an internuclear distance $r_{\mathrm{NH}}=1.04 \AA$. The constant, $-\mu_{\mathrm{o}}$, is the magnetic permittivity of vacuum, $h$ is Planck's constant, $\gamma_{X}$ is the magnetogyric ratio of spin $X$, and $r_{A B}$ is the distance between nuclei $A$ and $B$. The residual dipolar splitting between spins $A$ and $B$ equals

$$
D^{A B}=D_{\max }^{A B}\left\langle P_{2}(\cos \zeta)\right\rangle
$$

with $P_{2}(x)=\left(3 x^{2}-1\right) / 2$. Note that some texts differ in the definition of the dipolar coupling, $D_{A B}$, by a factor two.

If the molecule is rigid, the orientation of the internuclear vector, $r_{A B}$, in an arbitrary molecular coordinate system can be described by the angles $\alpha_{x}, \alpha_{y}$, and $\alpha_{z}$ between the vector and the $x, y$, and $z$ axis of the coordinate system. The $P_{2}(\cos \zeta)$ term can be expressed as

$$
\left\langle P_{2}(\cos \zeta)\right\rangle=\sum_{i, j=\{x, y, z\}} S_{i j} \cos \alpha_{i} \cos \alpha_{j}
$$

where $S$ is a traceless symmetric $3 \times 3$ matrix, commonly referred to as the Saupe matrix, the Saupe order matrix, or simply the order matrix.

If the structure of the molecule is known, i.e., $\cos \alpha_{i}$ factors in equation (2) are known, the five independent elements of $S$ generally can be solved provided that dipolar couplings for at least five internuclear vectors are available. However, if any pair of internuclear vectors is parallel, and for other special cases such as a set that includes three mutually orthogonal interactions, more measured couplings are required. For macromolecules, many more dipolar couplings are frequently measured, and $S$ is overdetermined. The Saupe matrix elements are then best determined using singular value decomposition. ${ }^{23}$

The order matrix is real and symmetric, and it therefore is always possible to define a molecular axis system where $S$ becomes diagonal. In a number of applications it can be advantageous to work in this principal axis frame, where $D^{A B}$ now can be expressed in terms of the polar coordinates of the $A-B$ internuclear vector:

$$
\begin{aligned}
D^{A B}(\theta, \phi)= & \frac{3}{2} D_{\max }^{A B}\left[\cos ^{2} \theta A_{z z}+\sin ^{2} \theta \cos ^{2} \phi A_{x x}\right. \\
& \left.+\sin ^{2} \theta \sin ^{2} \phi A_{y y}\right]
\end{aligned}
$$

where $A$ is the diagonalized alignment tensor, with $\left|A_{z z}\right|>$ $\left|A_{y y}\right|>\left|A_{x x}\right|$. This frequently is rewritten as

$$
D^{A B}(\theta, \phi)=D_{\max }^{A B}\left[P_{2}(\cos \theta) A_{a}+\frac{3}{4} A_{r} \sin ^{2} \theta \cos 2 \phi\right]
$$

where $A_{a}=3 A_{z z} / 2$ is the axial component of the alignment tensor, and $A_{r}=\left(A_{x x}-A_{y y}\right)$ is referred to as its rhombic component.

Note that the maximum value for $A_{a}$ equals one when the $z$ axis of the principal alignment tensor is fully aligned with the static field. In practice, the dilute liquid crystal work discussed in this article concerns $A_{a}$ values on the order of $10^{-3}$. Equation (3b) is sometimes rewritten as

$$
D^{A B}(\theta, \phi)=D_{a}^{A B}\left[\left(3 \cos ^{2} \theta-1\right)+\frac{3}{2} R \sin ^{2} \theta \cos 2 \phi\right]
$$

where $D_{a}^{A B}=1 / 2 A_{a} D_{\max }^{A B}$ is referred to as the magnitude of the dipolar coupling tensor, frequently normalized to the $\mathrm{N}-\mathrm{H}$ dipolar interaction, and $R=A_{r} / A_{a}$ is the rhombicity. Note that $0 \leq R \leq 2 / 3$.

\subsection{Estimate for Alignment Tensor in Case of Unknown Structure}

When working with a molecule of unknown structure, the above-described singular value decomposition approach for determining the order matrix is not applicable. However, as briefly discussed below, a reasonable estimate for the principal components of the alignment tensor can be obtained from the range and distribution of observed dipolar couplings.

First, it is convenient to normalize all observed one-bond and two-bond dipolar couplings to, for example, the $\mathrm{N}-\mathrm{H}$ dipolar coupling, by multiplying the observed $P-Q$ dipolar coupling by $\left(\gamma_{\mathrm{N}} \gamma_{\mathrm{H}} r_{P Q}^{3}\right) /\left(\gamma_{P} \gamma_{Q} r_{\mathrm{NH}}^{3}\right)$. Empirically determined optimum scaling factors are listed in Table 1. In the absence of measurement error in the dipolar couplings, the bond vector with the largest absolute value for the normalized dipolar coupling provides a lower limit for $2 D_{a}^{\mathrm{NH}}$ in equation (3c). Similarly, an estimate for the rhombicity $R$ in equation (3c) can be obtained from the dipolar coupling value, $D_{\text {opposite }}$, with the other extreme value (negative for $D_{a}^{\mathrm{NH}}>0$; positive for $D_{a}^{\mathrm{NH}}<0$ ). The value of $R$ itself, defined in this manner, is always positive and follows from $D_{\text {opposite }}=-D_{a}^{\mathrm{NH}}[1+$ $3 / 2 R]$, or

$$
R=-\frac{2}{3}\left[1+\left(\frac{D_{\text {opposite }}}{D_{a}^{\mathrm{NH}}}\right)\right]
$$

This approach only uses the extreme values of the distribution of observed dipolar couplings. A more robust approach plots the histogram of the entire ensemble of normalized dipolar couplings. ${ }^{24}$ Figure 1 shows an example of such histograms for the two globular domains of $\mathrm{Ca}^{2+}$-ligated calmodulin. The histograms have been compiled from nearly complete sets of ${ }^{1} D_{\mathrm{NH}},{ }^{1} D_{\mathrm{C} \alpha \mathrm{H} \alpha},{ }^{1} D_{\mathrm{C}^{\prime} \mathrm{N}},{ }^{2} D_{\mathrm{C}^{\prime} \mathrm{H} \alpha}$ and ${ }^{1} D_{\mathrm{C}^{\prime} \mathrm{C} \alpha}$ couplings, recorded in a medium of filamentous phage $P f l$.

Table 1 Magnitude of dipolar couplings relative to ${ }^{1} D_{\mathrm{NH}}$

\begin{tabular}{lll}
\hline & NMR $^{\mathrm{a}}$ & X-ray $^{\mathrm{b}}$ \\
\hline${ }^{1} D_{\mathrm{C} \alpha \mathrm{H} \alpha}$ & 2.08 & 2.02 \\
${ }^{1} D_{\mathrm{C} \alpha \mathrm{C}^{\prime}}$ & 0.198 & 0.198 \\
${ }^{1} D_{\mathrm{C}^{\prime} \mathrm{N}}$ & 0.120 & 0.121 \\
${ }^{2} D_{\mathrm{C}^{\prime} \mathrm{HN}}$ & 0.300 & 0.319 \\
${ }^{1} D_{\mathrm{CH} 3}$ & 0.628 & 0.628 \\
\hline
\end{tabular}

${ }^{a}$ Values that result in the lowest energy NMR structure of ubiquitin and yield best fit to ${ }^{13} \mathrm{C}^{\prime} \mathrm{CSA} .{ }^{74}$

${ }^{\mathrm{b}}$ Optimized by fitting experimental couplings to the ubiquitin X-ray structure. ${ }^{16}$ 


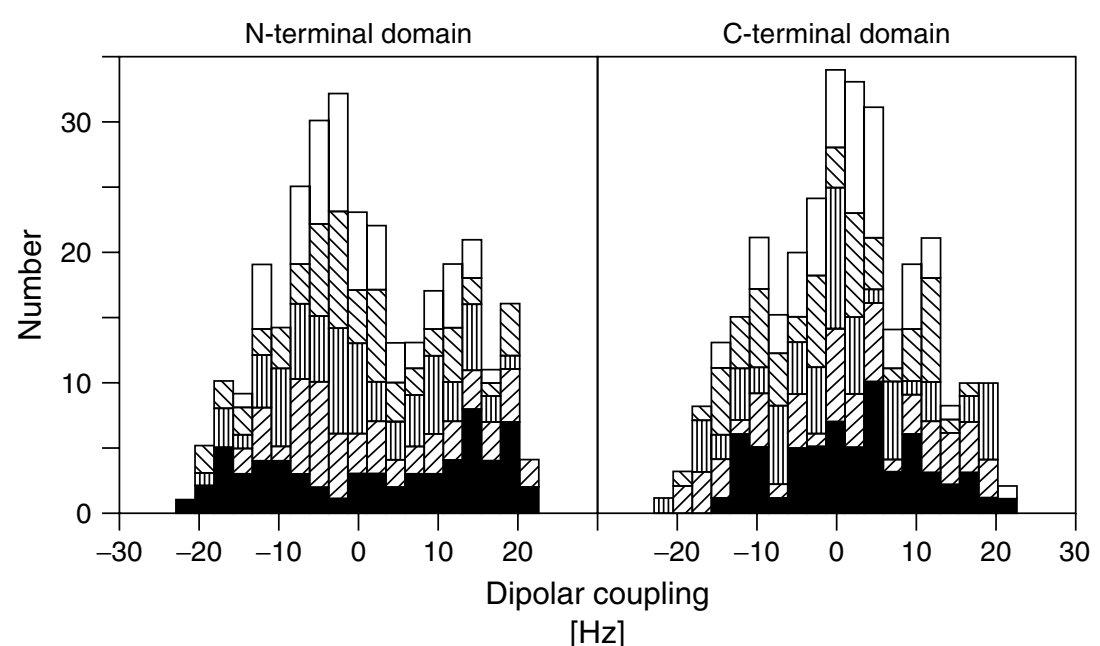

Figure 1 Histogram of normalized dipolar couplings measured in the $\mathrm{N}$ - and $\mathrm{C}$-terminal domains of $\mathrm{Ca}^{2+}$-ligated calmodulin, dissolved in a liquid crystalline medium containing $15 \mathrm{mg} \mathrm{Pf} 1 / \mathrm{ml}$ and $10 \mathrm{mM} \mathrm{KCl}$. Dipolar couplings are normalized relative to the one-bond ${ }^{15} \mathrm{~N}-{ }^{1} \mathrm{H}$ interaction. SVD fits to the crystal structure and the use of a freely floating alignment tensor during structure calculation ${ }^{77}$ both yield $D_{\mathrm{a}}^{\mathrm{NH}}=11.0 \mathrm{~Hz} ; R=0.38$ and $D_{\mathrm{a}}^{\mathrm{NH}}=10.1 \mathrm{~Hz} ; R=0.66$ for the $\mathrm{N}$ - and C-terminal domains, respectively. Note that even while the two histograms cover the same width, they have considerably different asymmetries (rhombicity, R). A dearth of bond vectors parallel to the z-axis of the alignment tensor causes the absence of dipolar couplings $>22 \mathrm{~Hz}$ for the $\mathrm{N}$-terminal domain. Different types of dipolar couplings are shaded separately in the histograms: $\left.{ }^{1} D_{\mathrm{NH}}-\operatorname{solid} ;{ }^{1} D_{\mathrm{C} \alpha \mathrm{H} \alpha}-\operatorname{shaded}(/ / I) ;{ }^{1} D_{\mathrm{C}^{\prime} \mathrm{N}}-\operatorname{shaded}(\backslash \backslash \backslash) ;{ }^{1} D_{\mathrm{C}^{\prime} \mathrm{C} \alpha}-\operatorname{shaded}(\mid \|)\right) ;{ }^{2} D_{\mathrm{C}^{\prime} \mathrm{H} \alpha}-\operatorname{blank}$

It can be shown that for a uniform distribution of bond vectors, such a histogram will resemble the solid state powder pattern observed for chemical shift anisotropy. ${ }^{25}$ In the present case, the singularities in the powder pattern correspond to $D_{z z}^{\mathrm{NH}}, D_{x x}^{\mathrm{NH}}$ and $D_{y y}^{\mathrm{NH}}$, with the condition that $D_{z z}^{\mathrm{NH}}+D_{x x}^{\mathrm{NH}}+$ $D_{y y}^{\mathrm{NH}}=0$. The relation between the powder pattern singularities $D_{z z}^{\mathrm{NH}}, D_{x x}^{\mathrm{NH}}, D_{y y}^{\mathrm{NH}}$ and the parameters $D_{a}^{\mathrm{NH}}$ and $R$ is given by: ${ }^{24}$

$$
\begin{aligned}
& D_{z z}^{\mathrm{NH}}=2 D_{a}^{\mathrm{NH}} \\
& D_{y y}^{\mathrm{NH}}=-D_{a}^{\mathrm{NH}}(1+1.5 R) \\
& D_{x x}^{\mathrm{NH}}=-D_{a}^{\mathrm{NH}}(1-1.5 R)
\end{aligned}
$$

If outliers appear to be present in the histogram, it is worth while checking the origin of these extreme dipolar couplings. They may correspond to ${ }^{1} D_{\mathrm{C}^{\prime} \mathrm{N}},{ }^{2} D_{\mathrm{C}^{\prime} \mathrm{H} \alpha}$ or ${ }^{1} D_{\mathrm{C}^{\prime} \mathrm{C} \alpha}$ couplings derived from weak or partially overlapping correlations, and critical visual inspection of the raw data may be needed before including them.

\section{MEASUREMENT OF DIPOLAR COUPLINGS}

To date, measurement of dipolar couplings has focused primarily on one-bond interactions, which as a result of their known internuclear distance, are readily interpreted in terms of orientation. Also, they are generally the easiest to measure. Two-bond interactions also have the benefit of a fixed internuclear distance, but owing to their larger separation, they can be more difficult to measure at the same level of relative accuracy. New analysis schemes promise to make measurement of ${ }^{1} \mathrm{H}-{ }^{1} \mathrm{H}$ dipolar couplings more straightforward, and they may also become popular as structural restraints.
Except for geminal ${ }^{1} \mathrm{H}-{ }^{1} \mathrm{H}$ interactions and pairs of protons in structural elements of fixed geometry, the interproton distance is an additional parameter influencing the dipolar coupling. This therefore results in a less direct relation between the value of the coupling and the orientation of the corresponding vector. However, structure calculation programs such as $\mathrm{CNS}^{\mathbf{2 6}}$ can readily deal with this additional complexity. ${ }^{27}$

So far, most measurements of dipolar couplings have focused on the one-bond and two-bond couplings along the polypeptide backbone in proteins (Figure 2), but dipolar couplings within and between sidechains are becoming increasingly used. Below, some of the most widely used techniques for measuring the various types of couplings are briefly discussed, with particular emphasis on ${ }^{15} \mathrm{~N}-{ }^{13} \mathrm{C}$ labeled proteins. With the exception of couplings between protons separated by more than three bonds, where the $J$ coupling is usually negligible, the coupling observed in the liquid crystalline phase represents the sum of the scalar and dipolar contributions. Considerable variation in the scalar couplings frequently exists,

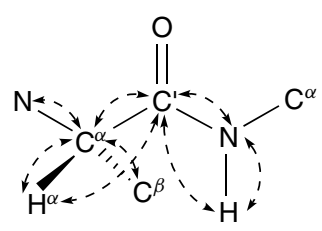

Figure 2 Protein backbone fragment, with dipolar interactions that can readily be measured marked by dashed lines. Although $D_{\mathrm{NC} \alpha}$ can be measured experimentally, its relative accuracy tends to be lower, and for trans-peptide bonds its normalized value is very similar to that of ${ }^{1} D_{\mathrm{C}^{\prime} \mathrm{C} \alpha}$ of the preceding residue. ${ }^{1} D_{\mathrm{C} \alpha \mathrm{C} \beta}$ is most useful in perdeuterated proteins, where no value for ${ }^{1} D_{\mathrm{C} \alpha \mathrm{H} \alpha}$ can be measured and where the slow transverse relaxation of the deuterated ${ }^{13} \mathrm{C}^{\alpha}$ permits its accurate measurement 
making it necessary to measure the couplings both in the isotropic and aligned environment.

Two conceptually different approaches can be used for measurement of $J$ or dipolar splittings. First, the splitting can be measured directly from a two- or three-dimensional spectrum recorded in the absence of decoupling of the interaction of interest. Frequently, an E.COSY ${ }^{28} \mathrm{~S}^{3} \mathrm{E}^{\mathbf{2 9}}$ or IPAP version $^{\mathbf{3 0}}$ of the multi-dimensional experiment is used in order to reduce overlap. Second, in a so-called quantitative- $J$ correlation experiment, ${ }^{\mathbf{3 1}}$ the size of the splitting can be derived from the intensity of a given resonance relative to that of a reference.

\subsection{Accuracy of Measured Splitting}

For splittings measured from relative peak displacements in the multi-dimensional NMR spectrum, the accuracy of a peak position is directly proportional to its signal-to-noise ratio, and inversely related to its line width. Although the accuracy also depends on the method used for peak position determination and on the digital resolution, a reasonable approximation for the root-mean-square uncertainty, $\Delta J$, in a measured splitting for a well resolved, undistorted, pure phase or pure antiphase doublet is given by:

$$
\Delta J=\frac{L W}{S N}
$$

where $L W$ is the line width at half height (in the dimension where the splitting is being measured), and $S N$ is the signalto-noise ratio. Note that equation (6) provides a lower limit for the true accuracy of the measurement, because other distortions such as partial overlap or imperfect phasing can contribute to the error in a reproducible manner.

\subsection{Measurement of ${ }^{1} J_{\mathrm{HN}}$}

One-bond ${ }^{1} D_{\mathrm{NH}}$ dipolar coupling were the first ones to be measured in a weakly oriented protein, simply by recording a ${ }^{1} \mathrm{H}-{ }^{15} \mathrm{~N}$ HSQC spectrum without the regular ${ }^{1} \mathrm{H} 180^{\circ}$ decoupling pulse applied at the midpoint of the $t_{1}$ evolution period. Transverse relaxation is considerably slower in the ${ }^{15} \mathrm{~N}$ dimension compared to the ${ }^{1} \mathrm{H}$ dimension, and measurement in the indirect ${ }^{15} \mathrm{~N}$ dimension is therefore preferred over measurement in the ${ }^{1} \mathrm{H}$ dimension. However, the spectral crowding doubles when HSQC spectra are recorded without decoupling, typically resulting in an unacceptable degree of resonance overlap.

A conceptually very simple method for measuring ${ }^{1} J_{\mathrm{NH}}$ separates the $\mathrm{F}_{1}$-coupled HSQC into two separate subspectra, containing only the upfield or downfield components of the $\mathrm{F}_{1}{ }^{15} \mathrm{~N}-\left\{{ }^{1} \mathrm{H}\right\}$ doublets. This is done by interleaving two experiments: one where the $\mathrm{F}_{1}{ }^{15} \mathrm{~N}-\left\{{ }^{1} \mathrm{H}\right\}$ doublets are in phase, and one where they are exactly antiphase. The sum of the antiphase and the in-phase spectra yields a spectrum containing only the downfield components, whereas the difference yields the upfield components (Figure 3). This approach is referred to as IPAP (for in-phase and anti-phase) ${ }^{\mathbf{3 2}}$ and is easily extended to higher dimensionality experiments.

A small region of the two-dimensional ${ }^{1} \mathrm{H}-{ }^{15} \mathrm{~N}$ IPAPHSQC spectrum of calmodulin is shown in Figure 3. Isotropic ${ }^{1} \mathrm{H}-{ }^{15} \mathrm{~N} J$ splittings are ca. $93 \mathrm{~Hz}$, and the large deviations

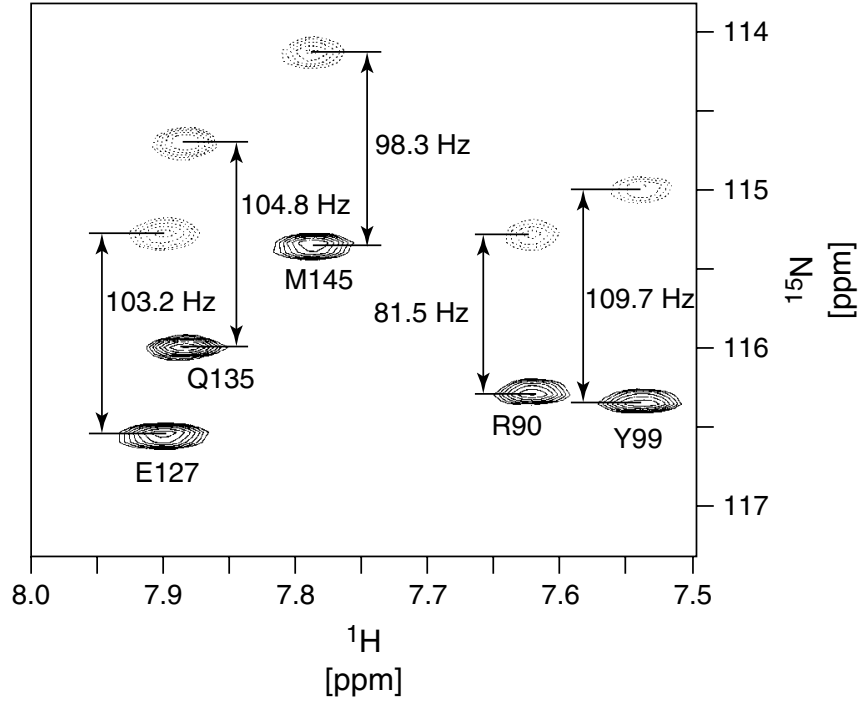

Figure 3 Small region of the IPAP ${ }^{15} \mathrm{~N}-{ }^{1} \mathrm{H}$ HSQC spectrum of $\mathrm{Ca}^{2+}$-ligated calmodulin in PfI medium. The solid and dashed contours correspond to the downfield and upfield ${ }^{15} \mathrm{~N}-\left\{{ }^{1} \mathrm{H}\right\}$ doublet components, which are separated into two two-dimensional subspectra. These subspectra are superimposed in this plot for visual simplicity, as none of the resonances overlap one another in the displayed region of the spectrum. The size of the splittings are marked in the figure and correspond to $\left|{ }^{1} J_{\mathrm{NH}}+{ }^{1} D_{\mathrm{NH}}\right|$

from this value correspond to the dipolar contributions to these splittings. So, both the sign and the magnitude of the dipolar coupling are obtained from such spectra.

\subsection{Measurement of ${ }^{1} J_{\mathrm{C}^{\prime} \mathrm{C} \alpha}$}

Due to the longer internuclear distance, and the lower product of the gyromagnetic ratios involved, the ${ }^{1} D_{\mathrm{C}^{\prime} \mathrm{C} \alpha}$ coupling is inherently five times smaller than the one-bond ${ }^{1} \mathrm{H}-{ }^{15} \mathrm{~N}$ coupling (Table 1). In order to obtain meaningful information, it therefore is necessary to measure this coupling at high accuracy. There are two ways to measure ${ }^{1} J_{\mathrm{C}^{\prime} \mathrm{C} \alpha}$ : observation of the $\mathrm{C}^{\alpha}$ or of the $\mathrm{C}^{\prime}$ resonance in the absence of decoupling the non-observed spin. For protonated proteins at moderate field strengths $(\leq 14 \mathrm{~T})$, the $\mathrm{C}^{\prime} T_{2}$ is invariably much longer than the $\mathrm{C}^{\alpha} T_{2}$, favoring detection of $\mathrm{C}^{\prime}$ for most accurate measurement of ${ }^{1} J_{\mathrm{C}^{\prime} \mathrm{C} \alpha}$. For perdeuterated proteins, detection of ${ }^{13} \mathrm{C}^{\alpha}$ in the presence of ${ }^{2} \mathrm{H}$ and ${ }^{13} \mathrm{C}^{\beta}$ decoupling yields narrower ${ }^{13} \mathrm{C}$ resonances, particularly at higher fields.

Usually, the most accurate method for measurement of ${ }^{1} J_{\mathrm{C}^{\prime} \mathrm{C} \alpha}$ is to simply record a 3D HNCO spectrum where the $\mathrm{C}^{\alpha}$ pulse, normally applied at the midpoint of the ${ }^{13} \mathrm{C}^{\prime}$ evolution period, is omitted. ${ }^{\mathbf{3 3}}$ The HNCO experiment yields among the best resolved triple resonance spectra and the two-fold increase in the number of resonances relative to the ${ }^{13} \mathrm{C}^{\alpha}$ decoupled spectrum is generally not much of a problem. The ${ }^{13} \mathrm{C}^{\prime}$ transverse relaxation rate is dominated by its chemical shift anisotropy, and ${ }^{13} \mathrm{C}^{\prime}$ line widths at $600 \mathrm{MHz}$ are about $30 \%$ larger than at $500 \mathrm{MHz}{ }^{1} \mathrm{H}$ frequency. It is therefore preferable to measure the ${ }^{1} J_{\mathrm{C}^{\prime} \mathrm{C} \alpha}$ splitting at a relatively low magnetic field strength. 


\subsection{Measurement of ${ }^{1} J_{\mathrm{C}^{\prime} \mathrm{N}}$ and ${ }^{2} J_{\mathrm{C}^{\prime} \mathrm{HN}}$}

Dipolar couplings across the peptide bond between ${ }^{15} \mathrm{~N}_{i}$ and ${ }^{13} \mathrm{C}_{i-1}^{\prime}$ are inherently 8.3 times weaker than ${ }^{1} D_{\mathrm{NH}}$ (Table 1 ). Therefore, accuracy of their measurement is critical for making optimal use of these small couplings in structure determination. Owing to the favorable relaxation properties of ${ }^{15} \mathrm{~N}$, especially when selecting the downfield ${ }^{15} \mathrm{~N}-\left\{{ }^{1} \mathrm{H}\right\}$ doublet component, the ${ }^{1} J_{\mathrm{C}^{\prime} \mathrm{N}}$ coupling is detected through the ${ }^{15} \mathrm{~N}$ and not the ${ }^{13} \mathrm{C}^{\prime}$ nucleus. The simplest method just records a twodimensional $\mathrm{HSQC}$ on the ${ }^{13} \mathrm{C} /{ }^{15} \mathrm{~N}$ doubly labeled protein, and inserts a ${ }^{13} \mathrm{C}^{\alpha}$ but not a ${ }^{13} \mathrm{C}^{\prime} 180^{\circ}$ decoupling pulse at the mid-point of ${ }^{15} \mathrm{~N}$ evolution. ${ }^{34}$ The resulting doublet in the ${ }^{15} \mathrm{~N}$ dimension corresponds to ${ }^{1} J_{\mathrm{C}^{\prime} \mathrm{N}}$. Interestingly, the detected amide proton is also coupled to ${ }^{13} \mathrm{C}^{\prime}$ (which has not changed its spin state during or after ${ }^{15} \mathrm{~N}$ evolution). Therefore, the two doublet components are also displaced relative to one another by the ${ }^{2} J_{\mathrm{C}^{\prime} \mathrm{HN}}$ coupling in the ${ }^{1} \mathrm{H}^{\mathrm{N}}$ dimension. Although normally this splitting would be very difficult to resolve because the ${ }^{1} \mathrm{H}^{\mathrm{N}}$ line width is typically much larger than the ${ }^{2} J_{\mathrm{C}^{\prime} \mathrm{HN}}$ coupling, the E.COSY principle ${ }^{28}$ effectively separates the two ${ }^{1} \mathrm{H}^{\mathrm{N}}-\left\{{ }^{13} \mathrm{C}^{\prime}\right\}$ doublet components (Figure 4).

An interesting alternative approach, based on the principle of quantitative $J$ correlation, derives ${ }^{1} J_{\mathrm{C}^{\prime} \mathrm{N}}$ from the relative intensities of two interleaved 3D TROSY-HNCO spectra. ${ }^{35}$ In one experiment (reference spectrum) the pulse scheme uses optimal one-bond ${ }^{15} \mathrm{~N}-\left\{{ }^{13} \mathrm{C}^{\prime}\right\}$ dephasing of $33 \mathrm{~ms}$, whereas in the second experiment (attenuated spectrum) the dephasing delay is set near the null condition $(66 \mathrm{~ms})$. Any deviation from ${ }^{1} J_{\mathrm{C}^{\prime} \mathrm{N}}+{ }^{1} D_{\mathrm{C}^{\prime} \mathrm{N}}=(66 \mathrm{~ms})^{-1}$ results in non-zero intensity in the second spectrum. The relative intensities of these two spectra then give a very precise measure for ${ }^{1} J_{\mathrm{C}^{\prime} \mathrm{N}}$, and permit

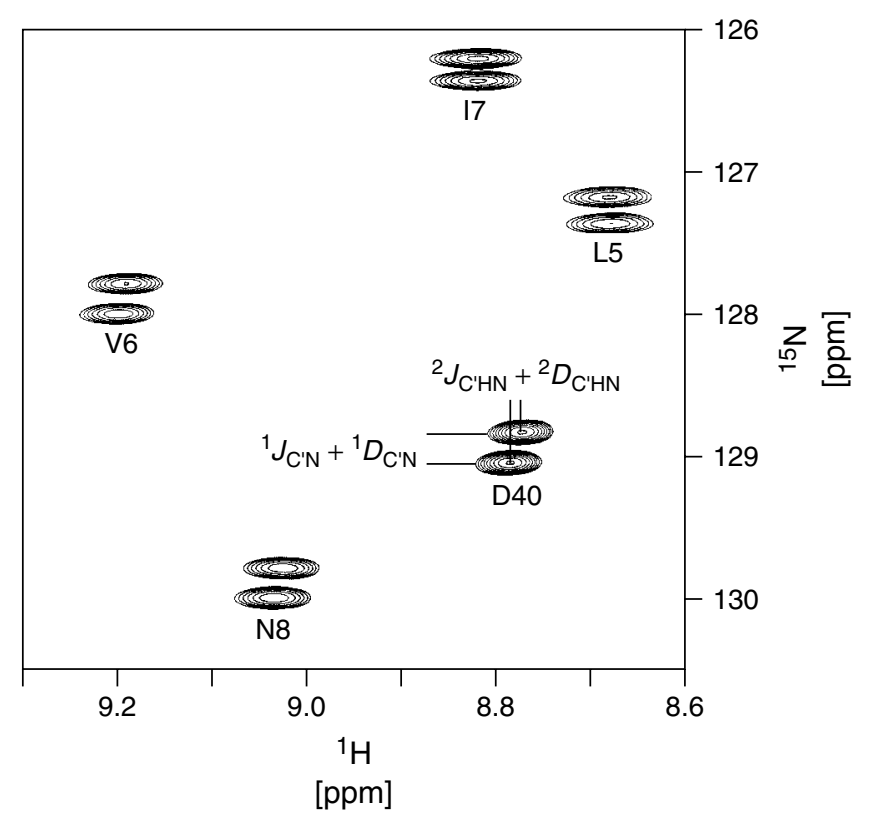

Figure 4 Small region of the ${ }^{15} \mathrm{~N}-{ }^{1} \mathrm{H}$ HSQC spectrum of the first immunoglobulin binding domain of protein $\mathrm{G}$, recorded in the absence of ${ }^{13} \mathrm{C}^{\prime}$ decoupling in Pfl liquid crystal $\left(11 \mathrm{mg} \mathrm{ml}^{-1}\right)$. The vertical and horizontal displacements within each doublet correspond to $\left|J_{\mathrm{C}^{\prime} \mathrm{N}}+{ }^{1} D_{\mathrm{C}^{\prime} \mathrm{N}}\right|$ and $\left.\right|^{2} J_{\mathrm{C}^{\prime} \mathrm{HN}}+{ }^{2} D_{\mathrm{C}^{\prime} \mathrm{HN}} \mid$, respectively. Doublets are labeled by their one-letter residue type and number
${ }^{1} D_{\mathrm{C}^{\prime} \mathrm{N}}$ to be derived at an accuracy that is a fraction of a hertz, provided the signal to noise in the reference spectrum exceeds $20: 1$.

\subsection{Measurement of ${ }^{1} J_{\mathrm{C} \alpha \mathrm{H} \alpha}$ and ${ }^{1} J_{\mathrm{C} \alpha \mathrm{C} \beta}$}

Although, in principle, ${ }^{1} J_{\mathrm{C} \alpha \mathrm{H} \alpha}$ can be measured from a ${ }^{1} \mathrm{H}-{ }^{13} \mathrm{C}$ two-dimensional correlation spectrum, recorded in the absence of heteronuclear decoupling in either the $F_{1}$ or $F_{2}$ dimension, the increase in spectral crowding frequently leads to unacceptable overlap for all but the smallest proteins. In practice, therefore, ${ }^{1} J_{\mathrm{C} \alpha \mathrm{H} \alpha}$ is most commonly measured either from a series of $J_{\mathrm{CH}}$-modulated HSQC spectra, simultaneous to measurement of sidechain $J_{\mathrm{CH}}$ couplings (see below), or from a $\mathrm{H}^{\alpha}$-coupled $\mathrm{HN}(\mathrm{CO}) \mathrm{CA}$ or $(\mathrm{HA}) \mathrm{CA}(\mathrm{CO}) \mathrm{NH}$ spectrum. This latter experiment makes it easy to decouple the ${ }^{1} J_{\mathrm{C} \alpha \mathrm{C} \beta}$ coupling by using a constant-time $\mathrm{C}^{\alpha}$ evolution period, and is therefore preferred when dealing with small or medium-size proteins. Its pulse sequence is identical to a scheme previously used for measuring relaxation interference between the ${ }^{13} \mathrm{C}^{\alpha}$ CSA tensor and the ${ }^{13} \mathrm{C}^{\alpha}-{ }^{1} \mathrm{H}^{\alpha}$ dipolar interaction, ${ }^{36}$ but now it is the splitting itself rather than the difference in doublet intensities that is the focus of the measurement.

In perdeuterated proteins, ${ }^{1} J_{\mathrm{C} \alpha \mathrm{H} \alpha}$ is inaccessible, but the long ${ }^{13} \mathrm{C}^{\alpha}$ transverse relaxation time permits measurement of ${ }^{1} J_{\mathrm{C} \alpha \mathrm{C} \beta}$ from a regular HNCA experiment, recorded in the presence of ${ }^{2} \mathrm{H}$ decoupling during ${ }^{13} \mathrm{C}^{\alpha}$ evolution. ${ }^{37}$

\subsection{Measurement of Sidechain ${ }^{1} J_{\mathrm{CH}}$ Couplings}

To date, most attention has focused on measurement of backbone-related dipolar couplings. Nevertheless, dipolar couplings also are starting to become used for studying sidechain conformations. However, a significant fraction of the sidechains may be subject to rotameric averaging, which reduces the dipolar coupling relative to a static, single conformation. If the smaller dipolar coupling is imposed during structure calculation as if it were rigid, this can result in distorted sidechain conformations with high energy. A way around this is to impose the observed dipolar coupling as a lower bound for the true dipolar coupling. ${ }^{\mathbf{3 8}}$ An even better way would be to evaluate for each site separately what the degree of sidechain mobility is by conducting the requisite relaxation experiments.

For small and medium-size proteins, an accurate way for extracting both backbone ${ }^{1} J_{\mathrm{C} \alpha \mathrm{H} \alpha}$ and sidechain ${ }^{1} J_{\mathrm{CH}}$ couplings is the recording of a set of CT-HSQC spectra, in which the ${ }^{1} \mathrm{H}$ $180^{\circ}$ decoupling pulse is systematically shifted. The intensity modulation pattern observed in the fully decoupled CT-HSQC spectrum then provides an accurate measure for ${ }^{1} J_{\mathrm{CH}}$. For methylene groups, the modulation frequency corresponds to the sum of the two ${ }^{1} J_{\mathrm{CH}}$ couplings. Methyl group intensities are modulated by ${ }^{1} J_{\mathrm{CH}}$ and by $3 \times{ }^{1} J_{\mathrm{CH}}$, with amplitudes of the modulation having a ratio of approximately $1: 3$. As a result of the rapid three-fold rotation about the three-fold symmetry axis of the methyl group, the ${ }^{1} D_{\mathrm{CH}}$ dipolar coupling is scaled down by -0.31 relative to its static value. ${ }^{39}$ Figure 5 compares the ${ }^{1} D_{\mathrm{CH}}$ and ${ }^{1} D_{\mathrm{CC}}$ dipolar couplings for methyl groups in ubiquitin. The tight correlation seen in this figure testifies to the precision at which these dipolar couplings can be measured. As suggested by this figure, for structure calculation 


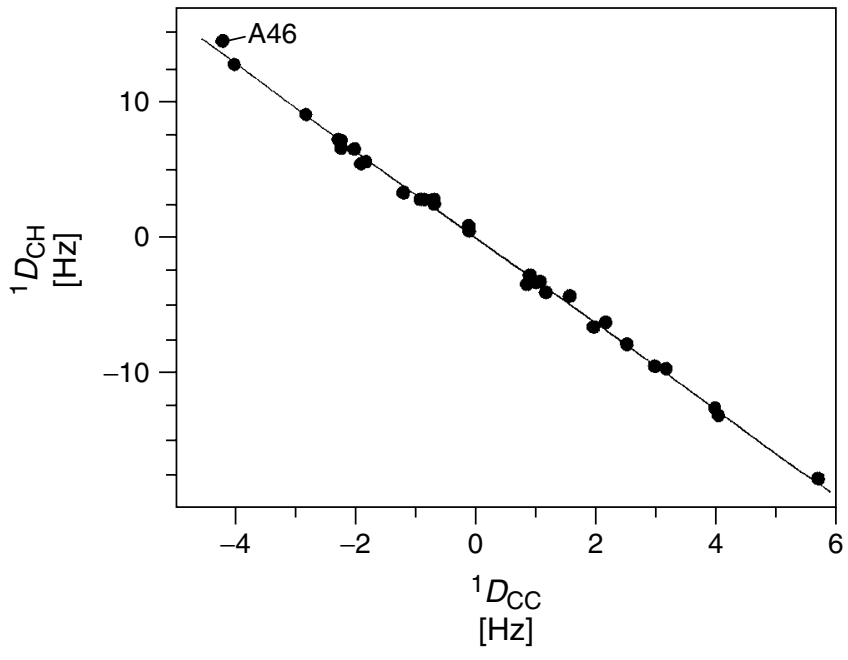

Figure 5 Plot of ${ }^{1} D_{\mathrm{CH}}$ versus ${ }^{1} D_{\mathrm{CC}}$ residual dipolar couplings of methyl groups in the protein ubiquitin, dissolved in a liquid crystalline medium containing $5 \%(\mathrm{w} / \mathrm{v})$ bicelles. The solid line represents the best fit of the data by linear regression, with a slope of $-3.17 \pm 0.03$. The outlier, labeled A46, corresponds to an alanine residue with a positive $\phi$ angle, for which there is a steric clash between the methyl group and a backbone carbonyl oxygen. (Reprinted, with permission, from Ottiger et al. ${ }^{\mathbf{3 9}}$ )

purposes the methyl group ${ }^{1} D_{\mathrm{CH}}$ value may be converted into ${ }^{1} D_{\mathrm{CC}}$, thereby restraining the $\mathrm{C}-\mathrm{C}\left(\mathrm{H}_{3}\right)$ orientation.

For larger proteins, the lower sensitivity and increased spectral overlap in a CT-HSQC spectrum frequently result in a relatively small fraction of residues for which the ${ }^{1} J_{\mathrm{CH}}$ coupling can be measured. As an alternative, spectra recorded with $\mathrm{CCH}-\mathrm{COSY}$ or $\mathrm{HCCH}-\mathrm{COSY}$, without decoupling in the detected dimension may be used for measurement of $J_{\mathrm{CH}}$. An additional benefit of this approach is that for the case of non-equivalent methylene protons, both $J_{\mathrm{CH}}$ couplings can be measured separately. In favorable cases, the ${ }^{1} \mathrm{H}-{ }^{1} \mathrm{H}$ multiplet structure in such spectra also permits measurement of ${ }^{2} J_{\mathrm{HH}} \cdot{ }^{40}$

\subsection{Measurement of ${ }^{\mathbf{1}} \mathrm{H}-{ }^{\mathbf{1}} \mathrm{H}$ Couplings}

The history of measuring ${ }^{1} \mathrm{H}-{ }^{1} \mathrm{H} J$ couplings is particularly rich and has been reviewed in numerous places. The E.COSY approach, applied in either a homo- or heteronuclear manner has proven particularly useful in this respect. ${ }^{28}$ However, few of these methods are suitable for measurement of ${ }^{1} \mathrm{H}-{ }^{1} \mathrm{H}$ dipolar couplings. When oriented, ${ }^{1} \mathrm{H}-{ }^{1} \mathrm{H}$ multiplet structure tends to become very complex as a result of the multitude of coupling partners any given proton experiences. Fortunately, in heteronuclear type E.COSY spectra this does not pose a serious problem, and the utility of triple resonance experiments for measurement of both the sign and magnitude of intraresidue and sequential $J_{\mathrm{H} \alpha \mathrm{HN}}$ couplings has been demonstrated in oriented media. ${ }^{\mathbf{4 1}}$ Nevertheless, with some exceptions, ${ }^{\mathbf{4 2 , 4 3}}$ such experiments tend to be less suitable for measurement of dipolar couplings that are spatially proximate but far apart in the covalent bond network. Alternatively, the popular HNHA experiment can be used for measuring ${ }^{1} \mathrm{H}-{ }^{1} \mathrm{H}$ dipolar couplings that involve an amide proton. ${ }^{\mathbf{6}, 44}$ In this experiment, the magnitude of $\left|J_{\mathrm{HH}}+D_{\mathrm{HH}}\right|$ is derived from the diagonal to cross peak intensity ratio. So, except for the intraresidue
$\mathrm{H}^{\mathrm{N}-} \mathrm{H}^{\alpha}$ interaction, where there also is a significant scalar contribution to the interaction, the sign of $D_{\mathrm{HH}}$ is not available from this experiment.

\section{ALIGNMENT MEDIA FOR MACROMOLECULES}

A prerequisite for studying macromolecules in a liquid crystalline phase is that the order imposed on the macromolecule is very small, typically less than 0.002 . The order of the liquid crystal particles themselves is invariably much higher, in the $0.5-0.85$ range. Clearly, for achieving such a weak solute alignment in the strongly oriented liquid crystalline medium, interaction between the macromolecule and the liquid crystal must be very weak. The medium must also be aqueous, such that the proteins or nucleic acids remain in their natural environment. An efficient way to satisfy these conditions is the use of a very dilute $(\leq 10 \%(\mathrm{w} / \mathrm{v}))$ lyotropic nematic liquid crystalline suspension, where the nematogenic unit itself is a large particle. In a liquid crystalline phase, the weak steric and electrostatic interactions between liquid crystal particles cause their cooperative alignment. There is a lower limit for the nematogen concentration, $c_{\mathrm{n}}$, required to form a stable liquid crystalline phase. If the sample is diluted below this threshold, it separates into an isotropic phase with concentration $c_{i}$ and a nematic phase, with the $c_{\mathrm{n}}$ being 5-20\% higher than $c_{i}$. The aspect ratio of the nematogenic particle is a critical determinant for the lowest concentration at which liquid crystalline ordering occurs. The time needed for formation of a homogeneous liquid crystalline phase strongly depends on the nematogen concentration, and can be very long for concentrated samples, but also for samples that are close to the threshold concentration, where separation between microscopic aligned and isotropic domains takes place.

All liquid crystalline media that have proven to be useful for weak ordering of proteins and nucleic acids consist of large (>1000 ̊), water-soluble particles, with relatively low surface charge densities $\left(\leq 0.5 \mathrm{e} \mathrm{nm}^{-2}\right)$. The interaction between the liquid crystal particles results in increased macroscopic viscosity relative to pure water. However, rotational diffusion of the macromolecule itself is virtually unchanged, unless it has a weak affinity for the liquid crystal particle. In the latter case, the degree of order typically is much too large and therefore is of little practical interest. For all lyotropic liquid crystals discussed below, the degree of order imposed on the solute can be adjusted by changing the concentration of the liquid crystal itself over the range where it yields an ordered phase. There is not yet a single, ideal liquid crystal. For example, phospholipases without inhibitors cannot be studied in bicelles as they break down the phospholipids; active proteases can destroy phage-based liquid crystals; other proteins may stick to phospholipids, or the surface charge distribution of the liquid crystal may cause too strong an electrostatic interaction with the solute. Also, liquid crystals require some degree of surface charge to keep their order and may fail to remain liquid crystalline at high salt concentrations. Below, several of the most widely used systems are briefly discussed.

\subsection{Bicelles}

Bicelles were the first liquid crystalline medium used for weak alignment of proteins and DNA. ${ }^{\mathbf{7 , 8}}$ These are planar 
bilayered particles that usually consist of regular saturated phospholipids. Most commonly, a mixture of dimyristoyl phosphatidyl choline (DMPC) and dihexanoyl phosphatidyl choline (DHPC) is used. DMPC makes up the bilayer, which constitutes the plane of the bicelle, and the DHPC detergent protects the long DMPC alkyl chains at the rims of the bilayer from exposure to solvent. Bicellar liquid crystals were originally developed by Prestegard, Sanders and co-workers for the purpose of studying lipophilic molecules, anchored in these highly ordered membranes. ${ }^{\mathbf{4 5}, \mathbf{4 6}}$ The DMPC/DHPC combination was found to be particularly robust. When raising the temperature above $25^{\circ} \mathrm{C}$, the system switches from isotropic to a nematic liquid crystalline phase. ${ }^{47}$ This temperature corresponds to the melting temperature of DMPC, i.e., the temperature at which the saturated alkane chains of DMPC melt from a crystalline or gel state, in which the alkane chains are all trans, to a flexible, liquid crystalline phase. Near this transition temperature, the sample has high macroscopic viscosity and tends to be slightly opaque. Below $25^{\circ} \mathrm{C}$ the solution remains clear and unordered and both small angle neutron scattering and NMR diffusion experiments indicate the bicelles to be disk-shaped bilayers in this unordered phase. ${ }^{\mathbf{4 8 , 4 9}}$

The phase diagram of the DMPC/DHPC mixture is very complex and depends on a large number of variables, including the absolute concentration, the molar ratio [DMPC]/[DHPC] $=$ $q$, temperature, ionic strength, and on the presence of charged amphiphilic molecules such as sodium dodecyl sulfate (SDS) or cetyl trimethylammonium bromide (CTAB). Bicelles were long believed to remain disk-shaped above the DMPC melting temperature, with a diameter determined by $q$. However, the diameters predicted by such a model are incompatible with the very dilute concentrations (down to $\leq 2 \%$ (v/v)) at which the sample remains liquid crystalline. Recent tracer diffusion obstruction and neutron scattering data indicate the liquid crystalline phase to consist of highly porous bilayers, presumably with the detergent covering the rims of the pores. ${ }^{49,50}$

Intrinsically, DMPC/DHPC bicelles can form a liquid crystalline phase over the temperature range of $25-45^{\circ} \mathrm{C}$. However, near the lower end of this range $\left(25-30{ }^{\circ} \mathrm{C}\right)$, dilute samples tend to be unstable and they frequently separate into an aligned and an isotropic phase. This can be prevented by addition of a small molar fraction (typically 1-3\%, relative to DMPC) of charged amphiphiles, such as CTAB (positive) or SDS (negative). ${ }^{\mathbf{5 1}}$ Alternatively, charged phospholipids such as dimyristoyl phosphatidylserine (negative), or dimyristoyl trimethylammonium propane (positive) can be used. Charging the bicelles in this manner helps prevent phase separation and widens the part of the phase diagram over which the liquid crystalline phase remains stable. The electrostatic potential repels and attracts oppositely charged groups on the solute, and generally causes a change in both the orientation and magnitude of the alignment tensor. This can be a highly beneficial side effect, as it permits measurement of dipolar couplings under multiple orientations of the protein (Figure 6). ${ }^{\mathbf{5 2}}$ Usually, this change in orientation is approximately linear with charge. So, if more than two different samples are prepared in this manner, they simply yield alignment tensors that are linear combinations of one another. ${ }^{53}$

Regular phospholipids are subject to acid- and basecatalyzed hydrolysis of the carboxyester linkage between the

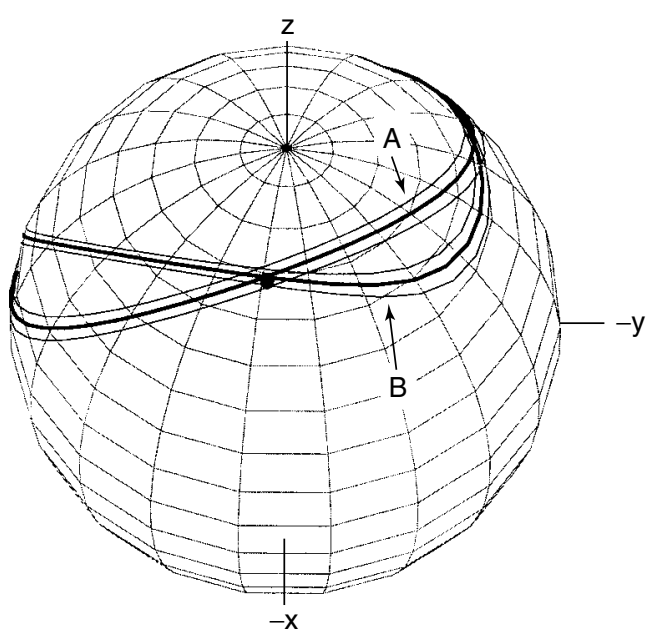

Figure 6 Orientations of the $\mathrm{Gln}^{40} \mathrm{~N}-\mathrm{H}$ vector in ubiquitin compatible with the ${ }^{15} \mathrm{~N}-{ }^{1} \mathrm{H}$ one bond dipolar coupling measured in undoped bicelles (band A) and in positively charged bicelles (band B). Orientations are given in the coordinate frame of the X-ray structure of this protein. Heavy lines correspond to the measured dipolar couplings; thinner lines correspond to orientations if $D_{\mathrm{NH}}$ is increased or decreased by $1 \mathrm{~Hz}$. The solid dot marks the orientation of the $\mathrm{N}-\mathrm{H}$ vector when the protons are model-built into the crystal structure, assuming $\mathrm{H}^{\mathrm{N}}$ is located on the line bisecting the $\mathrm{C}^{\prime}-\mathrm{N}-\mathrm{C}^{\alpha}$ angle. (Adapted, with permission, from Ramirez and $\mathrm{Bax}^{\mathbf{5 2}}$ )

alkyl chain and the glycerol backbone. The rate of hydrolysis is minimal at $\mathrm{pH} 6.5$, but rapidly increases once the $\mathrm{pH}$ differs by more than one unit from this value. ${ }^{54}$ Use of ether lipids, where the carboxyester bond is replaced by an ether linker can prevent this hydrolysis and such samples can be stable over a very wide range of $\mathrm{pH}^{\mathbf{5 5 , 5 6}}$ for periods of several years.

The temperature range over which the bicelles are liquid crystalline can be extended either by introducing a small fraction of mono-unsaturated DMPC, which lowers the melting temperature of the alkane chain, ${ }^{\mathbf{5 4}}$ or by using shorter alkane chains. Use of shorter alkane chain phospholipids, such as dilauroyl PC (12-C chains) $)^{57}$ or ditridecanoyl PC (13-C chains $)^{\mathbf{5 5}}$ lowers the transition temperature by about 12 and $6^{\circ} \mathrm{C}$, respectively, and increases the upper temperature limit as well. However, the macroscopic viscosity of these solutions below the transition temperature tends to be much higher than for DMPC/DHPC bicelles, such that preparation of the sample can be more cumbersome, particularly when using a microcell.

\subsection{Filamentous Phage}

Use of a liquid crystalline solution consisting of filamentous phages for aligning macromolecules was introduced in 1998., ${ }^{\mathbf{9}, 58}$ Clore et al. used a medium based on the filamentous phage $f d$, and also demonstrated the potential of tobacco mosaic virus (TMV). The Pardi group demonstrated the utility of the phage $P f 1$, which is similar to $f d$, but which at a contour length of ca. $2 \mu \mathrm{m}$ is nearly twice as long. Both phages have a diameter of $6.5 \mathrm{~nm}$, and are quite rigid, with a persistence length of ca. $1 \mu \mathrm{m}$. As a result of the high aspect ratios of these particles, solutions can remain liquid crystalline down to very low concentrations, as low as a 
few $\mathrm{mg} \mathrm{ml}^{-1}$. Although it was suggested that liquid crystallinity is independent of ionic strength, we find this to be true only at higher phage concentrations, ${ }^{15}$ whereas at lower concentrations $\left(\sim 13 \mathrm{mg} \mathrm{ml}^{-1}\right.$ for $\left.P f 1\right)$ the degree of alignment becomes sensitive to ionic strength, and at very high salt concentrations the samples become isotropic. In contrast to $f d, P f 1$ solutions tend to remain homogeneous when their concentration is lowered below the nematic threshold concentration, $c_{\mathrm{n}}$. However, below this threshold, the degree of both phage and solute alignment becomes a function of the magnetic field strength, characteristic of a so-called paranematic phase. ${ }^{15}$

The negative surface charge (ca. $0.5 e \mathrm{~nm}^{-2}$ ) is necessary to maintain a uniform suspension of these large particles. When lowering the $\mathrm{pH}$ below 6 , partial protonation of the glutamate and aspartyl sidechains of the phage coat protein reduces the net charge of the virus particle and the phage precipitates. However, $f d$ has been shown to be soluble and liquid crystalline when the $\mathrm{pH}$ is reduced further, to ca. $3 .{ }^{59}$

As highlighted by Clore et al. for an immunoglobulinbinding domain of Streptococcal protein G, the alignment tensor in the phage medium can be very different from that in the bicelle medium. ${ }^{\mathbf{1 0}}$ This is advantageous as it permits measurement of dipolar couplings relative to two independent axis systems, thereby resolving much of the degeneracy that occurs in interpreting a dipolar coupling in terms of two orientational variables, $\theta$ and $\phi$ in equation $(3 \mathrm{c})$. The phage particles are aligned with their long axis parallel to the magnetic field. Therefore, if the alignment of solute macromolecules were caused exclusively by steric interaction, the alignment tensor would be very similar to that in the bicelle medium. In practice, the alignment tensors in phage and bicelle media tend to be quite different as there usually are relatively strong electrostatic interactions between the phage and the solute (see also Section 5).

\subsection{Alkyl-poly(ethylene glycol) Based Media}

A lyotropic liquid crystal consisting of a mixture of alkylpoly(ethylene glycol) and hexanol is another very useful medium for protein and nucleic acid alignment. ${ }^{14}$ Alkylpoly(ethylene glycol) type compounds generally do not tend to bind to proteins, making this an attractive medium for a wide range of biological systems. In contrast to bicelles, the liquid crystal is not subject to hydrolysis and it can remain stable for years, provided the concentration of hexanol does not decrease as a result of slow evaporation. The medium remains liquid crystalline over a wide $\mathrm{pH}$ range and a considerable temperature range of $10-40{ }^{\circ} \mathrm{C}$, which is tunable by varying the type of alkyl-poly(ethylene glycol) and the chain length of the alcohol. ${ }^{14}$

The different alkyl-poly(ethylene glycol) compounds are referred to as $\mathrm{C} m \mathrm{E} n$, where $m$ is the number of carbons in the alkyl group, and $n$ is the number of ethylene glycol units. Typically, a hexanol to $\mathrm{C}_{12} \mathrm{E}_{5}$ molar ratio of 0.96 (5 wt.\% $\mathrm{C}_{12} \mathrm{E}_{5}$ in $\mathrm{H}_{2} \mathrm{O}$ ) is used yielding a particularly stable liquid crystal that is easy to prepare, but the medium is relatively tolerant to changes in the absolute and relative concentrations. $\mathrm{C}_{12} \mathrm{E}_{5}$ is commercially available at low cost. In many cases, the sample can simply be recovered from the liquid crystal by extensive dialysis.

\subsection{Other Liquid Crystals}

Ternary mixtures of cetylpyridinium chloride $(\mathrm{CPCl})$ or bromide ( $\mathrm{CPBr})$, hexanol, and $\mathrm{NaCl}$ or $\mathrm{NaBr}$ in water can form stable liquid crystalline phases over a wide range of conditions. Prosser et al. ${ }^{11}$ demonstrated that the $\mathrm{CPCl}$ version yielded alignment for the protein ubiquitin, and Barrientos et al. ${ }^{\mathbf{1 2}}$ described the use of the $\mathrm{CPBr}$ based medium. Remarkable differences were found between the $\mathrm{CPBr}$ and $\mathrm{CPCl}$ based liquid crystals, with $\mathrm{CPBr}$ being more suitable for low ionic strength measurements $(10-40 \mathrm{mM} \mathrm{NaBr})$ and the $\mathrm{CPCl}$ system requiring higher salt concentrations $(200-500 \mathrm{mM}$ $\mathrm{NaCl}){ }^{\mathbf{1 2}}$

Dilute suspensions of natural cellulose fibers, derived either from wood pulp or from filter paper by careful sulfuric acid hydrolysis, also can form a liquid crystalline phase suitable for macromolecular ordering. ${ }^{60}$ Although negatively charged, the net surface charge density is lower than for filamentous phage, making it a useful complement to the other media, mentioned above. However, the lower surface charge and the smaller aspect ratio relative to filamentous phage result in a higher minimal threshold concentration for liquid crystal formation.

A suspension of purple membrane (PM) fragments has also been shown suitable for inducing a weak degree of macromolecular order. ${ }^{\mathbf{5 3 , 6 1}}$ These fragments consist primarily $(75 \%(\mathrm{w} / \mathrm{w}))$ of bacteriorhodopsin, an integral membrane protein with seven transmembrane helices. The PM fragments have an average diameter of about $1 \mu \mathrm{m}$. This is sufficiently large that the total magnetic susceptibility anisotropy of such a particle causes it to align nearly $100 \%$ when placed in a strong magnetic field $(\geq 11 \mathrm{~T})$, with the membrane plane orthogonal to the direction of the magnetic field. ${ }^{62}$ So, in contrast to the bicelle, phage and $\mathrm{CPBr}$ systems, $\mathrm{PM}$ does not need to form a liquid crystalline phase for obtaining alignment.

The strong net negative surface charge of PM causes very weak transient binding of solute proteins that carry clusters of positively charged groups on their surfaces, and thereby can result in net alignment. ${ }^{\mathbf{5 3 , 6 1}}$ The electrostatic interactions are typically weak enough not to distort the structure of globular proteins. However, as is the case with all liquid crystal studies in this chapter, when studying flexible regions in a protein, care must be taken when interpreting the dipolar coupling data as the protein only transiently interacts with the liquid crystal. Interaction with the nematogen may be favored when the flexible region temporarily adopts a given shape, whereas other, non-binding conformations of the flexible region may not be sampled.

\subsection{Polyacrylamide Gel}

In order to prevent sedimentation, suspensions of large particles such as phages, bicelles, and cellulose require a repelling interaction, i.e., a net surface charge. These local electrostatic fields generally result in stronger solute alignment, which then may become too strong for facile dipolar coupling measurement. In other cases, solutes may cause the liquid crystal particles to aggregate and precipitate. For example, no stable liquid crystal has yet been described that is suitable for detergent-solubilized proteins. So, although the above mentioned liquid crystalline systems will cover the majority of systems of interest, there remain exceptions. 
Recently, a very useful alternative method for inducing weak alignment has been developed that is known as straininduced alignment in a gel (SAG). ${ }^{\mathbf{6 3 , 6 4}}$ This method relies on anisotropically compressed polyacrylamide gel, which forms an extremely stable and inert aqueous matrix for solute proteins, and even permits their study under partially or fully denaturing conditions. ${ }^{65}$ The gels may either be compressed or stretched in the axial direction, ${ }^{\mathbf{6 4}}$ with stretching generally yielding stronger alignment. ${ }^{\mathbf{6 6}}$ The robustness of such a medium makes it particularly useful for challenging applications such as detergent-solubilized systems. ${ }^{\mathbf{6 6}}$ The solutes may either be diffused into the gel, or they may be included in the medium before polymerization of the hydrogel is induced.

Compression of the gel can simply be achieved by first casting it in a narrow diameter $(3-3.5 \mathrm{~mm})$ cylinder. Then, after soaking in the solute of interest, it is transferred to a regular 4.2-mm inner diameter (ID) NMR sample tube, followed by axial compression by a susceptibility-matched plunger. Stretching can be achieved by radial compression, using a funnel-like device to squeeze a larger diameter gel $(5-7 \mathrm{~mm})$ into a regular 4.2-mm ID NMR sample tube. ${ }^{\mathbf{6}}$ Polyacrylamide gels appear to yield the most widely applicable method for inducing weak macromolecular alignment as the system is very inert and stable. Their principal disadvantage is a potential decrease in rotational diffusion rate of the solute, resulting in line broadening. ${ }^{64,67}$ This latter effect can be minimized by using gel concentrations as low as possible $(\leq 5 \%)$ combined with high degrees of stretching, and by using low cross-linking ratios $(\leq 1: 40)$.

\subsection{Use of Multiple Alignment Media}

As discussed in Section 5, solute alignment is defined by both steric and electrostatic interaction. So, by using nearly neutral liquid crystals, such as bicelles or the alkyl poly(ethylene-glycol) medium, and a charged medium such as phage or the cetyl-pyridinium based phase, a single molecule can be studied using two or more independent alignment tensors. As illustrated in Figure 6, this removes much of the degeneracy in the relation between dipolar coupling and internuclear vector orientation. ${ }^{\mathbf{5 2}}$ A dipolar coupling measured in a given medium defines the internuclear vector to be on one of two oppositely oriented cones. The alignment tensor in a second medium will generally be oriented differently relative to the molecular frame and a dipolar coupling defines the same vector to be situated on a different cone. The true internuclear vector orientation then must be located on one of the intersections between the two sets of cones (Figure 6). In the general case of a non-axially symmetric (rhombic) alignment tensor there are up to eight intersections. If a third, independent alignment tensor can be obtained, this degeneracy may be reduced to two-fold. The true orientation and its inverse can never be distinguished from measurements on a single dipolar interaction, and two-fold degeneracy is therefore the best that can be achieved for a single vector.

Besides changing the liquid crystal medium, the alignment tensor may also be altered by subtly changing the protein. For example, when the $\mathrm{pH}$ is altered such that the surface charge distribution becomes different, this will affect the alignment tensor in the charged liquid crystalline media. Similarly, if protein preparations are available with and without a Histag tail, their alignment tensors will generally be different, although not necessarily by a large amount. Also, for a protein with a His-tag, a relatively small change in $\mathrm{pH}$ from 6 to 7.5 can significantly alter the alignment tensor.

If one-bond dipolar couplings are measured for a set of non-collinear interactions in a fragment of known secondary structure, such as an $\alpha$-helix, there are four different ways to orient the fragment relative to the alignment tensor. Dipolar couplings measured in a second, independent medium, can lift this degeneracy, defining its orientation uniquely. ${ }^{68}$

\section{RELATION BETWEEN ALIGNMENT AND SHAPE}

Tjandra et al. demonstrated that in a bicelle medium the principal axes of the molecular alignment tensor closely coincide with those of the rotational diffusion tensor. , $^{69}$ This shows that in this nearly neutral medium, alignment is defined by the solute's shape. As mentioned above, the alignment tensor can be modified by adding a net charge to the bicelles, by doping them with either CTAB (+) or SDS $(-)$. This demonstrates that electrostatic interactions also play a role. In fact, for an oriented medium of strongly negatively charged, rod-shaped viral particles, or oriented PM fragments, electrostatic interactions usually dominate alignment of solute proteins.

A simple steric model has been proposed that quantitatively describes the relation between the solute's shape and its alignment in the type of lyotropic liquid crystals described in this article. ${ }^{70}$ So far, it has only been demonstrated for the case of (nearly) neutral particles, such as bicelles, but preliminary results indicate that the method can be extended to account for the effect of charge.

In the so-called steric-obstruction model, the solute sample can be simulated as a collection of randomly oriented, uniformly distributed molecules, from which the fraction that sterically clashes with the ordered array of liquid crystal particles is removed. For example, for a lamellar phase such as bicelles or $\mathrm{C}_{12} \mathrm{E}_{5}$, a larger fraction of molecules oriented orthogonal to the bilayer will be obstructed than molecules parallel to the surface, resulting in net ordering of the remaining, non-obstructed molecules. For each non-obstructed molecule a Saupe order matrix is calculated, using equation (2). Averaging of the Saupe matrices for all non-obstructed proteins than yields the sterically predicted alignment tensor. ${ }^{70}$ In an extension of this method that accounts also for the effect of electrostatics, different weighting factors are given to each of the non-obstructed solute molecules, depending on the Boltzmann factor calculated when taking the electrostatic potential into account (M. Zweckstetter, unpublished results).

Figure 7 shows the correlation between the ${ }^{15} \mathrm{~N}-{ }^{1} \mathrm{H}$ dipolar couplings measured for the Ig binding domain of Streptococcal protein $\mathrm{G}$, and that predicted from its $1.0 \AA$ crystal structure, using an alignment tensor that is not best-fitted to the data, but calculated on the basis of its shape. ${ }^{69}$ When ignoring electrostatics, the predicted alignment tensors for bicelle and phage media are very similar. However, the experimentally observed dipolar couplings in the two media are very different and, as expected, good agreement is only observed for the bicelle medium (Figure 7). When electrostatic terms are included in the calculations for the phage medium, the 

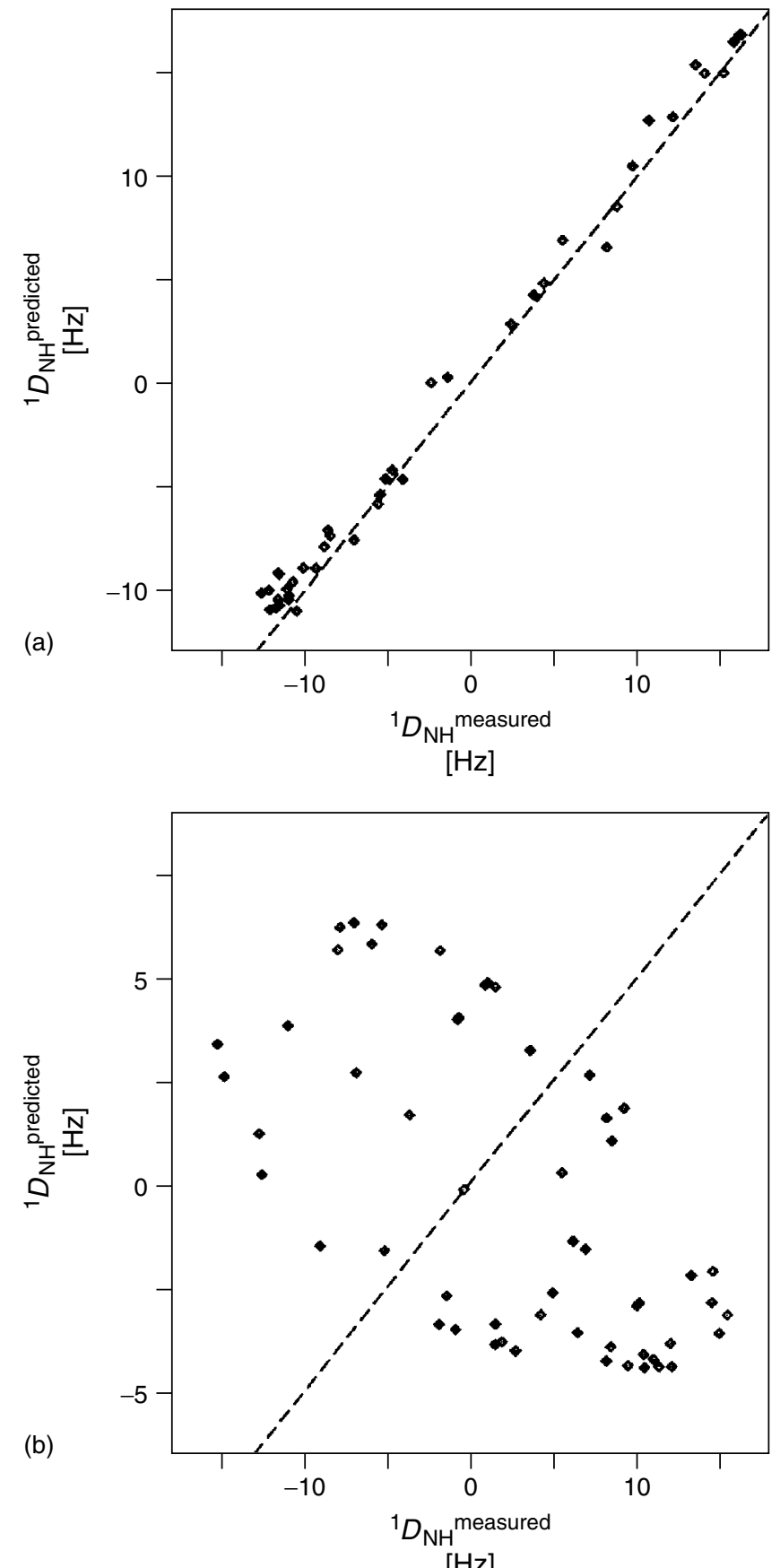

$[\mathrm{Hz}]$

Figure 7 Correlations between experimental ${ }^{1} D_{\mathrm{NH}}$ values and values calculated from the shape-predicted alignment tensor of the third immunoglobulin binding domain of protein $G$ in (a) $5 \%$ (w/v) bicelle medium, and (b) $28 \mathrm{mg} \mathrm{ml}^{-1} f d$ medium. Dashed lines correspond to $y=x$. The poor correlation in (b) indicates that in phage medium the protein alignment is dominated by electrostatic interactions, which are ignored in the alignment tensor prediction, and not by steric interaction. (Adapted, with permission, from Zweckstetter and $\mathrm{Bax}^{\mathbf{1 5}}$ )

agreement becomes nearly as good as for the neutral bicelle medium. Note that in contrast to the results of an SVD fit, the data shown in Figure 7 do not include any adjustable parameters. So, the alignment tensor orientation and magnitude are calculated on the basis of the protein's shape, and subsequently dipolar couplings are predicted on the basis of the orientation of the internuclear vector relative to this alignment tensor.

\section{STRUCTURE VALIDATION}

One particularly attractive feature of dipolar couplings is that they can be used in a very direct manner to evaluate the accuracy of any given structure: the more accurate the structure, the better the agreement is between experimental dipolar couplings and this structure. In order to evaluate this agreement, one first needs to determine the alignment tensor or Saupe matrix. As mentioned earlier, there are two conceptually different ways to do this. One can either predict the alignment on the basis of the molecule's shape and charge distribution, or one can determine the Saupe matrix elements of equation (2) simply by singular value decomposition. There are five independent elements in the Saupe matrix. If the number of experimentally measured independent (i.e., noncollinear) dipolar interactions is much larger than five, the SVD approach is preferred as it eliminates the effect of errors in the shape-predicted alignment tensor. Clearly, for effective validation it is necessary that the number of observables (dipolar couplings) is much larger than the number of variables in the SVD fit. If this condition is not met, corrections can be made for the significance of the correlation in terms of $F$ statistics. For proteins, typically the number of observed dipolar couplings is much larger than five, and the goodness of the fit provides a direct measure for the accuracy of the structure.

Figure 8 shows a typical example of the correlation between measured dipolar couplings and those predicted by two structures of the $\mathrm{C}$-terminal domain of $\mathrm{Ca}^{2+}$-calmodulin (after best fitting the Saupe matrix to the experimental couplings). The first correlation (Figure $8 \mathrm{a}$ ) is calculated by using the $1.0-\AA \mathrm{X}$ ray crystal structure of Paramecium tetraurelia calmodulin to fit the data measured for the highly homologous mammalian protein. It yields a Pearson's correlation coefficient, $R^{P}$, of 0.95 . The second structure was calculated on the basis of $\mathrm{N}-\mathrm{H}$, $\mathrm{C}^{\prime}-\mathrm{N}, \mathrm{H}^{\alpha}-\mathrm{C}^{\alpha}$ and $\mathrm{C}^{\prime}-\mathrm{C}^{\alpha}$ dipolar couplings, and the $\mathrm{H}^{\alpha}-\mathrm{C}^{\prime}$ couplings are best-fit to this structure. Clearly, the NMR structure $\left(R^{P}=0.98\right)$ predicts the experimental $\mathrm{H}^{\alpha}-\mathrm{C}^{\prime}$ couplings better than the X-ray structure, which is related to a slight rearrangement of relative helix orientations in solution relative to the crystalline state. ${ }^{71}$

When accurate experimental dipolar couplings are available, it is always advantageous to incorporate them into the structure calculation process. However, once this is done, they no longer are useful for validation purposes. A way around this is to compare the observed and predicted changes in chemical shift between isotropic and aligned samples for a type of nucleus with a well-defined chemical shift anisotropy. The first application of this idea was for the change in ${ }^{15} \mathrm{~N}$ chemical shift with field strength in a small protein-DNA complex, ${ }^{72}$ caused by the anisotropy of the magnetic susceptibility of this complex. Although the changes were minute $(\leq 5 \mathrm{ppb})$, a clear improvement in the correlation was observed upon incorporation of dipolar couplings in the structure calculation process. Subsequently, comparison of much larger ${ }^{13} \mathrm{C}^{\prime}$ chemical shift changes between a protein in isotropic and aligned bicellar medium also showed an excellent correlation with structure. ${ }^{\mathbf{7 3}}$ 


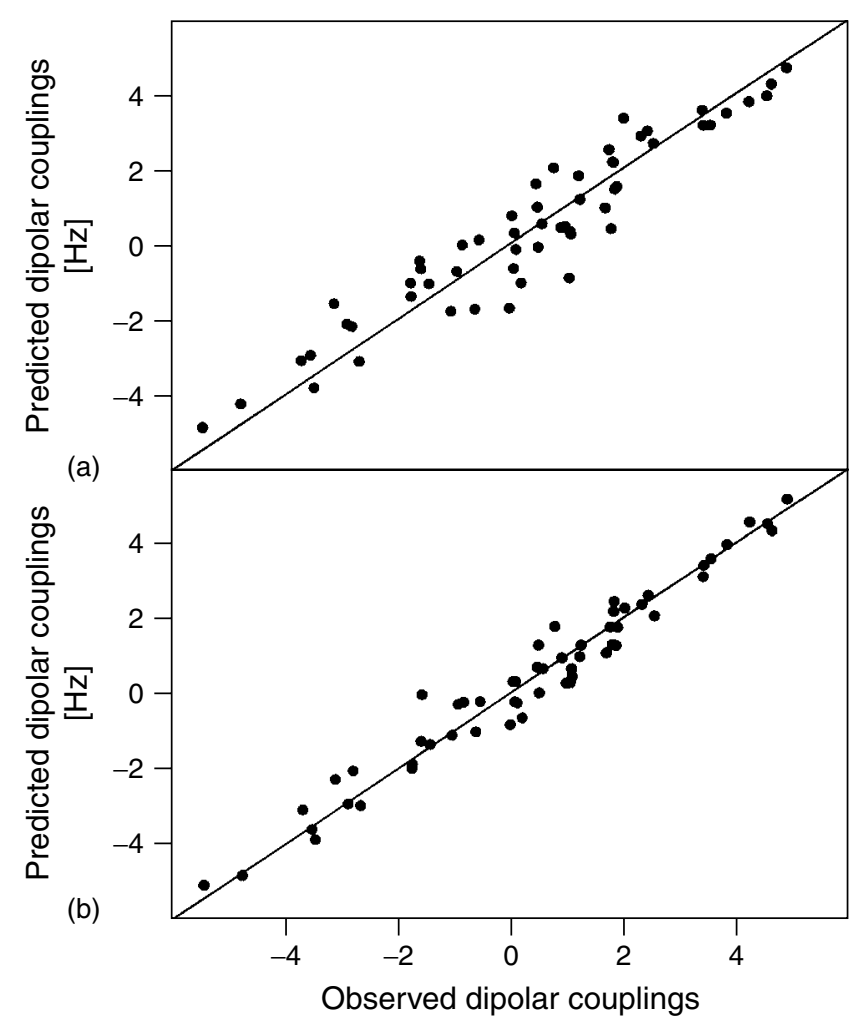

$[\mathrm{Hz}]$

Figure 8 Correlation between measured, normalized ${ }^{2} D_{\mathrm{C}^{\prime} \mathrm{H} \alpha}$ dipolar couplings and those predicted by the $1.0-\AA \mathrm{X}$-ray crystal structure (a), and by the NMR structures of the C-terminal domain of $\mathrm{Ca}^{2+}$ calmodulin, calculated without ${ }^{2} D_{\mathrm{C}^{\prime} \mathrm{H} \alpha}$ couplings (b). The slightly better fit to the NMR structure is caused by a small rearrangement of the relative helix orientations in solution relative to those observed in the crystalline state of this protein $^{71}$

\section{CONCLUDING REMARKS}

The introduction of methods for generating tunable, very weak alignment of macromolecules makes it possible to measure the one-bond and other short range dipolar couplings with a remarkable degree of accuracy. Even while the splittings are reduced by about three orders of magnitude relative to their static values, the narrow line widths and relatively high signal-to-noise ratio attainable in high resolution NMR more than compensate for the low degree of order. For example, one-bond ${ }^{15} \mathrm{~N}-{ }^{1} \mathrm{H}$ dipolar couplings typically can be measured with an accuracy of ca. $0.1 \mathrm{~Hz}$, whereas their range covers up to $50 \mathrm{~Hz}$. Even for the intrinsically much smaller interactions, such as the one-bond ${ }^{15} \mathrm{~N}-{ }^{13} \mathrm{C}^{\prime}$ dipolar coupling, the error typically is smaller by up to two orders of magnitude relative to the range of couplings observed. Similarly, chemical shift effects can be measured at a relative accuracy that rivals that attainable in solid state NMR, but the problem of resonance overlap is much less severe because isotropic shifts in solution are more effective at dispersing NMR spectra. Experimental correlations between ${ }^{13} \mathrm{C}^{\prime},{ }^{15} \mathrm{~N}$, and ${ }^{1} \mathrm{H}^{\mathrm{N}} \mathrm{CSA}$ tensors and secondary structure in proteins could be clearly established from such data. ${ }^{74}$

Dipolar couplings are not very sensitive to small amplitude oscillations about an average orientation. Conversely, relatively small discrepancies between observed and predicted magnitudes of dipolar couplings require rather large amplitude angular fluctuations if this discrepancy is entirely attributed to dynamic effects. ${ }^{\mathbf{7 5}, 76}$ Nevertheless, because averaging of the observed dipolar interactions is sensitive to motions that cover the entire time scale from milliseconds to femtoseconds, it can potentially provide a powerful complement for the study of dynamic processes.

Measurement of tensorial interactions in macromolecules is a new and rapidly expanding area in structural biology. It offers numerous new opportunities, including rapid determination of backbone folds, rapid structure determination, refinement of conventional NMR structures, and the study of relative orientations of components in multi-molecular complexes. It is likely that a further marriage between conventional solidstate NMR techniques and the weak alignment approach may expand these areas even further.

\section{RELATED ARTICLES IN VOLUMES 1-8}

Biological Macromolecules: Structure Determination in Solution, Volume 2; Liquid Crystals: General Considerations, Volume 4; Liquid Crystals: Mixed Magnetic Susceptibility Solvents, Volume 4; Two-Dimensional NMR of Molecules Oriented in Liquid Crystalline Phases, Volume 8.

\section{REFERENCES}

1. A. Saupe and G. Englert, Phys. Rev. Lett., 1963, 11, 462-464.

2. J. W. Emsley in 'Encyclopedia of NMR', eds D. M. Grant and R. K. Harris, Wiley: Chichester, 1996, Vol. 4, pp 2788-2799.

3. E. W. Bastiaan, C. Maclean, P. C. M. vanZijl, and A. A. BothnerBy, Annu. Rep. NMR Spectrosc., 1987, 19, 35-77.

4. A. A. Bothner-By, in 'Encyclopedia of NMR', eds D. M. Grant and R. K. Harris, Wiley: Chichester, 1996, Vol. 5, pp 2932-2938.

5. J. R. Tolman, J. M. Flanagan, M. A. Kennedy, and J. H. Prestegard, Proc. Natl. Acad. Sci. USA, 1995, 92, 9279-9283.

6. N. Tjandra, J. G. Omichinski, A. M. Gronenborn, G. M. Clore, and A. Bax, Nature Struct. Biol., 1997, 4, 732-738.

7. A. Bax and N. Tjandra, J. Biomol. NMR, 1997, 10, 289-292.

8. N. Tjandra and A. Bax, Science, 1997, 278, 1111-1114.

9. M. R. Hansen, L. Mueller, and A. Pardi, Nature Struct. Biol., 1998, 5, 1065-1074.

10. G. M. Clore, M. R. Starich, and A. M. Gronenborn, J. Am. Chem. Soc., 1998, 120, 10571-10572.

11. R. S. Prosser, J. A. Losonczi, and I. V. Shiyanovskaya, J. Am. Chem. Soc., 1998, 120, 11010-11011.

12. L. G. Barrientos, C. Dolan, and A. M. Gronenborn, J. Biomol. NMR, 2000, 16, 329-337.

13. K. Fleming, D. Gray, S. Prasannan, and S. Matthews, J. Am. Chem. Soc., 2000, 122, 5224-5225.

14. M. Ruckert and G. Otting, J. Am. Chem. Soc., 2000, 122, $7793-7797$.

15. M. Zweckstetter and A. Bax, J. Biomol. NMR, 2001, 20, 365-377.

16. M. Ottiger and A. Bax, J. Am. Chem. Soc., 1998, 120, $12334-12341$.

17. D. W. Yang, R. A. Venters, G. A. Mueller, W. Y. Choy, and L. E. Kay, J. Biomol. NMR, 1999, 14, 333-343.

18. P. Permi, P. R. Rosevear, and A. Annila, J. Biomol. NMR, 2000, 17, 43-54.

19. J. H. Prestegard, Nature Struct. Biol., 1998, 5, 517-522.

20. N. Tjandra, Struct. Fold. Des., 1999, 7, R205-R211. 
21. A. Bax, G. Kontaxis, and N. Tjandra, Methods Enzymol., 2001, 339, 127-174.

22. E. Brunner, Concepts Magn. Reson., 2001, 13, 238-259.

23. J. A. Losonczi, M. Andrec, M. W. F. Fischer, and J. H. Prestegard, J. Magn. Reson., 1999, 138, 334-342.

24. G. M. Clore, A. M. Gronenborn, and A. Bax, J. Magn. Reson., 1998, 133, 216-221.

25. D. M. Grant, in 'Encyclopedia of NMR', eds D. M. Grant and R. K. Harris, Wiley: Chichester, 1996, Vol. 2, pp 1298-1321.

26. A. T. Brunger, P. D. Adams, G. M. Clore, W. L. DeLano, P. Gros, R. W. Grosse-Kunstleve, J. S. Jiang, J. Kuszewski, M. Nilges, N. S. Pannu, R. J. Read, L. M. Rice, T. Simonson, and G. L. Warren, Acta Crystallogr. Sec. D-Biol. Crystallogr., 1998, 54, 905-921.

27. N. Tjandra, J. Marquardt, and G. M. Clore, J. Magn. Reson., 2000, 142, 393-396.

28. C. Griesinger, O. W. Sørensen, and R. R. Ernst, J. Magn. Reson., 1987, 75, 474-492.

29. A. Meissner, J. O. Duus, and O. W. Sorensen, J. Biomol. NMR, 1997, 10, 89-94.

30. M. Ottiger, F. Delaglio, and A. Bax, J. Magn. Reson., 1998, 131, 373-378.

31. A. Bax, G. W. Vuister, S. Grzesiek, F. Delaglio, A. C. Wang, R. Tschudin, and G. Zhu, Methods Enzymol., 1994, 239, 79-105.

32. M. Ottiger, F. Delaglio, and A. Bax, J. Magn. Reson., 1998, 131, 373-378.

33. S. Grzesiek and A. Bax, J. Magn. Reson., 1992, 96, 432-440.

34. F. Delaglio, D. A. Torchia, and A. Bax, J. Biomol. NMR, 1991, 1, 439-446.

35. J. J. Chou and A. Bax, J. Biomol. NMR, 2000, 18, 101-105.

36. N. Tjandra and A. Bax, J. Am. Chem. Soc., 1997, 119, 9576-9577.

37. J. Evenas, A. Mittermaier, D. W. Yang, and L. E. Kay, J. Am. Chem. Soc., 2001, 123, 2858-2864.

38. M. Ottiger, F. Delaglio, J. L. Marquardt, N. Tjandra, and A. Bax, J. Magn. Reson., 1998, 134, 365-369.

39. M. Ottiger and A. Bax, J. Am. Chem. Soc., 1999, 121, 4690-4695.

40. E. T. Olejniczak, R. P. Meadows, H. Wang, M. L. Cai, D. G. Nettesheim, and S. W. Fesik, J. Am. Chem. Soc., 1999, 121, 9249-9250.

41. M. L. Cai, H. Wang, E. T. Olejniczak, R. P. Meadows, A. H. Gunasekera, N. Xu, and S. W. Fesik, J. Magn. Reson., 1999, 139, 451-453.

42. G. Otting, M. Ruckert, M. H. Levitt, and A. Moshref, J. Biomol. NMR, 2000, 16, 343-346.

43. W. Peti and C. Griesinger, J. Am. Chem. Soc., 2000, 122, 3975-3976.

44. G. W. Vuister and A. Bax, J. Am. Chem. Soc., 1993, 115, $7772-7777$.

45. C. R. Sanders and J. H. Prestegard, Biophys. J., 1990, 58, $447-460$

46. C. R. Sanders and J. H. Prestegard, J. Am. Chem. Soc., 1991, 113, 1987-1996.

47. C. R. Sanders and J. P. Schwonek, Biochemistry, 1992, 31, 8898-8905.

48. P. A. Luchette, T. N. Vetman, R. S. Prosser, R. E. W. Hancock, M. P. Nieh, C. J. Glinka, S. Krueger, and J. Katsaras, Biochim. Biophys. Acta-Biomembranes, 2001, 1513, 83-94.

49. S. Gaemers and A. Bax, J. Am. Chem. Soc., 2001, in press.

50. M. P. Nieh, C. J. Glinka, S. Krueger, R. S. Prosser, and J. Katsaras, Langmuir, 2001, 17, 2629-2638.

51. J. A. Losonczi and J. H. Prestegard, J. Biomol. NMR, 1998, 12, 447-451.
52. B. E. Ramirez and A. Bax, J. Am. Chem. Soc., 1998, 120, 9106-9107.

53. J. Sass, F. Cordier, A. Hoffmann, A. Cousin, J. G. Omichinski, H. Lowen, and S. Grzesiek, J. Am. Chem. Soc., 1999, 121, 2047-2055.

54. M. Ottiger and A. Bax, J. Biomol. NMR, 1998, 12, 361-372.

55. M. Ottiger and A. Bax, J. Biomol. NMR, 1999, 13, 187-191.

56. S. Cavagnero, H. J. Dyson, and P. E. Wright, J. Biomol. NMR, 1999, 13, 387-391.

57. H. Wang, M. Eberstadt, E. T. Olejniczak, R. P. Meadows, and S. W. Fesik, J. Biomol. NMR, 1998, 12, 443-446.

58. M. R. Hansen, M. Rance, and A. Pardi, J. Am. Chem. Soc., 1998, 120, 11210-11211.

59. L. G. Barrientos, J. M. Louis, and A. M. Gronenborn, J. Magn. Reson., 2001, 149, 154-158.

60. K. Fleming, D. Gray, S. Prasannan, and S. Matthews, J. Am. Chem. Soc., 2000, 122, 5224-5225.

61. B. W. Koenig, J. S. Hu, M. Ottiger, S. Bose, R. W. Hendler, and A. Bax, J. Am. Chem. Soc., 1999, 121, 1385-1386.

62. B. A. Lewis, C. Rosenblatt, R. G. Griffin, J. Courtemanche, and J. Herzfeld, Biophys. J., 1985, 47, 143-150.

63. R. Tycko, F. J. Blanco, and Y. Ishii, J. Am. Chem. Soc., 2000, 122, 9340-9341.

64. H. J. Sass, G. Musco, S. J. Stahl, P. T. Wingfield, and S. Grzesiek, J. Biomol. NMR, 2000, 18, 303-309.

65. D. Shortle and M. S. Ackerman, Science, 2001, 293, 487-489.

66. J. J. Chou, S. Gaemers, B. Howder, J. M. Louis, and A. Bax, J. Biomol. NMR, 2001, 21, 377-382.

67. Y. Ishii, M. A. Markus, and R. Tycko, J. Biomol. NMR, 2001, 21, 141-151.

68. H. M. Al-Hashimi, H. Valafar, M. Terrell, E. R. Zartler, M. K. Eidsness, and J. H. Prestegard, J. Magn. Reson., 2000, 143, 402-406.

69. E. de Alba, J. L. Baber, and N. Tjandra, J. Am. Chem. Soc., 1999, 121, 4282-4283.

70. M. Zweckstetter and A. Bax, J. Am. Chem. Soc., 2000, 122, 3791-3792.

71. J. J. Chou, S. Li, C. B. Klee, and A. Bax, Nature Struct. Biol., 2001, 8, 990-997.

72. M. Ottiger, N. Tjandra, and A. Bax, J. Am. Chem. Soc., 1997, 119, 9825-9830.

73. G. Cornilescu, J. L. Marquardt, M. Ottiger, and A. Bax, J. Am. Chem. Soc., 1998, 120, 6836-6837.

74. G. Cornilescu and A. Bax, J. Am. Chem. Soc., 2000, 122, $10143-10154$.

75. J. R. Tolman, J. M. Flanagan, M. A. Kennedy, and J. H. Prestegard, Nature Struct. Biol., 1997, 4, 292-297.

76. J. Meiler, J. J. Prompers, W. Peti, C. Griesinger, and R. Bruschweiler, J. Am. Chem. Soc., 2001, 123, 6098-6107.

77. S. Moltke and S. Grzesiek, J. Biomol. NMR, 1999, 15, 77-82.

\section{Biographical Sketch}

Ad Bax, $b$ 1956. Ph.D. 1981 Applied Physics, Delft University of Technology, The Netherlands. Research associate, Colorado State University, 1982-83; National Institutes of Health, Visiting Scientist, NMR Section, Laboratory of Chemical Physics, NIDDK, 1983-88. National Institutes of Health, Chief, Biophysical NMR Spectroscopy Section, 1988-present. Approx. 300 publications. Current research interests: NMR and its applications in chemistry, biology and medicine. 